Meta

Journal des traducteurs

Translators' Journal

\title{
Essai de logico-syntaxe comparée : la traduction de la conjonction anglaise as
}

\section{Paul Jinot}

Volume 35, numéro 1, mars 1990

Actes du colloque international « La traduction proligère "

URI : https://id.erudit.org/iderudit/003264ar

DOI : https://doi.org/10.7202/003264ar

Aller au sommaire du numéro

Éditeur(s)

Les Presses de l'Université de Montréal

ISSN

0026-0452 (imprimé)

1492-1421 (numérique)

Découvrir la revue

Citer cet article

Jinot, P. (1990). Essai de logico-syntaxe comparée : la traduction de la conjonction anglaise as. Meta, 35(1), 162-176. https://doi.org/10.7202/003264ar d'utilisation que vous pouvez consulter en ligne. 


\section{ESSAI DE LOGICO-SYNTAXE COMPARÉE : LA TRADUCTION DE LA CONJONCTION ANGLAISE $A S$}

PAUL JINOT

Université Laurentienne, Sudbury, Canada

1. Dans les langues naturelles, à fonction de communication, tout se ramène en fin de compte à la sémantique. Toutefois, si l'on veut éviter la circularité, la sémantique ne peut être saisie (dans la mesure où elle se laisse saisir) que par l'entremise des processus qui l'informent. Mon propos est d'examiner, dans une perspective comparative, quelquesunes des valeurs qui caractérisent le sémantisme de la conjonction anglaise as. Pour des raisons pratiques, dans le cadre du présent exposé, mon analyse se limitera aux sens de as autres que comparatifs et aux constructions où la circonstancielle introduite par as suit la principale. Je qualifie le problème de «logico-syntaxique» parce que as est un mot grammatical - et non pas un mot plein, à la signification intrinsèque -, dont la fonction est de relier logiquement les articulations syntaxiques de la phrase, et dont le sémantisme ne se définit que par rapport aux éléments qu'il raccorde.

Le contexte linguistique dans lequel doit évoluer le français au Canada expose cette langue à une entropie croissante. Cette évolution est particulièrement dangereuse lorsqu'elle touche le niveau des structures logico-syntaxiques, le cour de la langue, celui qui - à travers les vents et marées des changements phonétiques, diachroniques et synchroniques (dialectes) ou des passades lexicales, reflets éphémères tant de l'irrésistible fuite en avant de la technologie que de la remise en question de plus en plus vigoureuse des modèles sociologiques traditionnels, lorsqu'il ne s'agit pas tout simplement du newspeak de tous ceux, publicitaires, politiciens, propagandistes, qui ont vocation de manipuler, par jargon interposé, les cordons de notre bourse ou le fil de nos pensées - assure la permanence et l'identité de la langue à travers les générations. Or, la traduction systématique de ce as par alors que, calque que pratiquent trop souvent les médias canadiens-français, est un exemple caractéristique de ce noyautage qui finit par vider une langue de sa substance vive. Mon propos est de passer en revue, dans les limites spécifiées ci-dessus, les principales valeurs de $a s$, et de montrer en quoi alors que peut s'identifier avec elles et à partir de quel point il convient d'invoquer d'autres équivalences.

\subsection{Le plan de la temporalité pure}

Comme c'est généralement le cas de l'anglicisme, il y a équivalence sémantique partielle entre as et le français alors que dans le cadre général de l'expression de la simultanéité. Partielle, car le registre aspectuel de as est beaucoup plus vaste que celui de alors que: il recoupe en effet, à une extrémité, celui de while, dans l'expression de la durée, mais aussi, à l'autre, celui de when, dans l'expression de la simultanéité ponctuelle. Or, alors que, dans son sens temporel, gouverne nécessairement une proposition d'aspect duratif, progressif ${ }^{1}$, et ne peut donc jamais traduire un as qui soit l'équivalent fonctionnel de when, comme dans l'exemple suivant:

(1) The crowd was beginning to disperse as the guest of honor arrived, 
dans lequel le verbe de la subordonnée exprime une action ponctuelle, plaquée sur la toile de fond progressive de la principale, rapport aspectuel étranger au sémantisme d'alors que.

L'exemple type d'une équivalence as-alors que serait sans doute celui-ci :

(2) Three dogs attacked and killed a Jacksonville, Fla., woman (today) as she fetched her paper. A bystander had to use a baseball bat to beat the dogs off as they dragged her body down the street.

(2) Il s'est fait mordre par un chien alors qu'il faisait du jogging dans le parc.

Notons ici que le français met plus naturellement la proposition subordonnée introduite par cet alors que temporel en tête de phrase:

(3) Alors qu'elle braconne sur les terres de Martin Chasserand, le hasard met Julia en présence du fils de celui-ci, Bruno...

De toute façon, ces exemples types sont des réalisations du modèle structural suivant:

(4) $\mathrm{X}$ happens / ed (ponctuel, perfectif) as $\mathrm{Y}$ goes on / went on = is / was going on (aspect progressif de la as-clause - forme progressive souvent non marquée, remplacée par forme simple - présent ou prétérit — polyvalente);

(4') événement $\mathrm{X}$ (survenir)... alors que (= à l'heure où, au moment où) telle (être) la situation / événement $\mathrm{Y}$ (survenir) ( $\mathrm{Y}$ duratif par rapport à $\mathrm{X}$ ponctuel)

qui illustre ce que Le Grand Robert de la langue française, dans un autre contexte il est vrai, appelle «l'incidence d'un événement instantané par rapport à un fait continu» ( $c f$. note 20 , a). Le rapport aspectuel entre les deux propositions s'établit comme suit:

principale: action ponctuelle,

- subordonnée introduite par as/alors que: toile de fond/arrière-plan duratif (si perspective passée, rapport logique marqué le plus souvent par le jeu [passé composé ou simple vs imparfait ou prétérit vs passé de la forme progressive (parfois masqué sous un prétérit aspectuellement neutre)].

Ce rapport logique se laisserait gloser ainsi ${ }^{2}$, par inversion des propositions, mais en respectant strictement la relation aspectuelle:

(5) $Y$ was going on when $X$ happened.

(5') Telle était la situation lorsque / quand tel événement s'est produit,

soit, pour les exemples (2-4) donnés plus haut:

(2') Il faisait tranquillement son jogging lorsque tout à coup il a été attaqué et mordu par un chien.

(2a) The woman was on her way to getting her paper when she was attacked and killed by a dog.

(3a') Elle braconnait sur les terres de M. C. lorsque le hasard la mit en présence... (après transposition au passé pour mieux dégager le rapport aspectuel).

En revanche, on dirait difficilement:

1a) L'invité d'honneur est arrivé lorsque la foule commençait à se disperser (?)

car ce lorsque, naturellement ponctuel, gouvernerait plutôt un temps d'aspect perfectif (passé simple ou passé composé : a commencé). L'emploi de l'imparfait dénote une action de type duratif, précisement conforme au modèle de (4'), si bien qu'on pourrait dire, au lieu de (1'): 
(1b') L'invité d'honneur est arrivé alors que la foule commençait à se disperser.

Le polysémantisme de as fait que l'anglais peut l'utiliser dans des constructions où les rapports aspectuels seraient incompatibles avec l'emploi du alors que temporel français.

1.2. Le cas type discuté ci-dessus entre en fait dans un cadre plus général, où l'opposition (aspect ponctuel du verbe de la proposition principale vs aspect duratif de la subordonnée introduite pas as) s'estompe, soit dans un sens (les deux propositions tendent plus ou moins vers le ponctuel - sans toutefois que la subordonnée l'atteigne totalement:

(6) He spilled his beer as he got up (- he spilled his beer when he got up);

si l'action de spilling est bien instantanée, celle de getting up, si brève soit-elle, conserve une certaine épaisseur qui pourrait se manifester, au besoin, par l'emploi de la forme progressive : «... as he was getting up»), soit dans l'autre :

(7) Cape Town march draws 20,000 as police remains out of sight.

Ici, les deux propositions s'inscrivent dans la durée, à l'intersection de as et while.

Advenant le cas limite de la coextension pure et simple dans la durée (as $=>$ while), l'équivalence as-alors que demeure donc possible. Toutefois, la voie n'est plus aussi directe que dans le cas type : en effet, comme je l'ai mentionné plus haut, le français préfère mettre la proposition introduite par alors que devant la principale et la séparer de celle-ci par une virgule, ce qui peut avoir pour effet de lui donner une valeur de communication que la as-clause, en position finale, n'aurait pas:

(8') Mardi, alors que l'un des membres du comité militaire au pouvoir descendait de son hélicoptère, les camions affrétés par la Croix-Rouge allemande (...) procédaient à des distributions de nourriture (...)

La position de alors que en tête de phrase, comme dans le cas de since/puisque ou comme, tend à faire de la proposition subordonnée le thème de la phrase, alors que l'opposition thème-propos ou information connue-information nouvelle se trouve neutralisée lorsque la subordonnée introduite par as / alors que suit la principale - ce qui expliquerait la popularité de as dans les titres ou les paragraphes d'introduction d'articles de journaux, où aucune information ne peut être supposée connue a priori. Certes, rien n'interdit à la proposition introduite par as, éventuellement précédée de just/even, de figurer en tête de phrase, comme en français :

(8) Tuesday, even as one of the members of the ruling military junta was stepping off his helicopter, people from the German Red Cross were busy handing out food supplies (...)

Mais le français a une raison autre que la thématisation pour préférer placer ce alors que temporel en tête de phrase: alors que, entre deux propositions décrivant des événements symétriques, prend naturellement son autre valeur, dominante en fait, qui est la valeur d'opposition ( $c f .1 .4$ ) : Jean travaillait alors que Pierre dormait (while - whereas), ou (Quand elle est entrée), Jean s'est levé alors que Pierre est resté assis (ibid.). En général, et surtout là où les événements juxtaposés sont dans un rapport explicite d'antonymie fonctionnelle (par ex., isomorphisme des prédicats, à un élément - éventuellement au niveau des traits sémantiques - près, comme dans les deux exemples précédents), le sens adversatif s'impose. Pour relier sans équivoque deux événements A et $\mathrm{B}$, duratifs, simultanés et logiquement indépendants 1'un de l'autre, il demeure préférable de faire appel à pendant que: (A pendant que $\mathrm{B}$ ), de même que l'anglais, dans pareil cas, serait porté à employer while plutôt que as. 
Notons enfin, pour mémoire, que :

i) si le sujet grammatical est le même, le français traduit as temporel (ainsi d'ailleurs que while et when) au moyen de en + participe présent; en revanche, dans la même situation, as, non plus que because, ne permet l'ellipse du sujet + auxiliaire devant le participe présent, ellipse parfaitement naturelle avec while (going...), when (leaving...), since (leaving...), par exemple;

ii) si la coextension se présente sous forme d'une réaction soutenue ou répétée à un stimulus soutenu ou répété (aspect progressif ou itératif), le français fait appel à $a u$ fur et à mesure que, ou encore à une transposition:

(...) il arrive souvent qu'un verbe anglais subordonné se rend plus naturellement en français par un substantif : (...)

- The natives opened out as he came up.

- Les indigènes s'écartèrent sur son passage ${ }^{3}$.

Il est vrai que nous nous trouvons ici à la limite du as strictement temporel et du as causal (voir plus bas 2.2);

(iii) Ia relation aspectuelle définie ci-dessus connaît en français une manifestation originale: l'expression du contexte général duratif peut être confiée à des temps tels que le plus-que-parfait (l'explosion s'est produite alors que l'avion avait atterri et roulait sur la piste : alors que se fait ici inchoatif: il marque alors le début de l'état qui constitue la toile de fond; as n'est pas compatible avec cette perspective, qu'il rendra plutôt à l'aide du participe présent ou de (after + participe présent) : ... Quelques heures plus tard, alors qu' il avait appris par la femme de son cousin Nappo que les gendarmes le cherchaient, il fonçait en pleine nuit chez d'autres amis...) ou la construction (venir de + inf.) (même chose en plus net encore). Comme nous l'avons déjà noté en (1.1), ce parallélisme de as/alors que temporel est effectivement très partiel et ne porte que sur les constructions les plus fondamentales.

1.3. L'exemple suivant illustre le cas type (was about to = variante de l'aspect progressif was going to), en même temps qu'il permet de dégager la spécificité de as par rapport à ses concurrents dans l'expression générale de la simultanéité :

(9a) A Soviet-built TU-22 bomber crewed by Libyans was shot down by the French last week, [...], as it was about to bomb the capital, in retaliation for a push into Libya.

L'aspect fondamentalement ponctuel de when le rend incompatible avec to be about to (en revanche, il serait parfaitement correct de dire when it crossed/strayed into Chadian airspace, cas où when $=>$ as soon as).

While est virtuellement possible, mais en fait introduit un contexte trop vague qui peut même aller jusqu'à la simple simultanéité de deux événements distincts, physiquement et logiquement autonomes, par exemple :

(9b) A Soviet-built TU-22 bomber [...], while the Security Council debated/was debating the Libya-Chad conflict.

À noter que as peut lui aussi s'employer dans ce dernier contexte:

(9c) A Soviet-built TU-22 bomber [...], as the Security Council debated/was debating the $[\ldots]$

La nuance qu'apporte as (tout comme alors que), au lieu de la simple coïncidence d'événements indépendants l'un de l'autre qu'exprime while (ou pendant que), c'est une 
réduction du contexte à l'essentiel ou, dans le cas de l'exemple précédent, la focalisation logique sur ce contexte en fonction de l'idée exprimée dans la principale (idée de paradoxe, d'ironie du sort, d'opportunité de la réunion de Conseil de Sécurité). C'est pourquoi il se prête naturellement au renforcement auquel j'ai fait allusion plus haut, au moyen de just/even (se démarquant en cela de when et de while), tout comme le français (a)lors (même) que (*lorsque même, *(ce)pendant même que, *tandis même que). As permet de resserrer le contexte, de le réduire aux éléments immédiatement pertinents de la simultanéité; c'est ce que j'appellerais un effet de zoom, que l'on va retrouver en fait à travers tout le sémantisme de as. C'est tout particulièrement le cas lorsque l'action décrite dans la principale met (ou, comme dans le cas de They almost scored in the final minute of play as the puck rolled across the goal-crease, mettrait) fin à l'événement-toile de fond décrit par la subordonnée (par ex. [2], peut-être [2'] selon que la morsure permet ou non au sujet de continuer son jogging, et [9a]). À la limite, ce zoom comprime tellement le contexte que les informations portées par les deux propositions s'interpénètrent et la complémentarité fait place à l'intégration:

(10a) This small South Texas town prepared today to bury the first of 19 victims of a school bus accident as investigators tried to determine why a truck ran a stop sign and rammed the bus into a water-filled pit.

L'intégration est telle et l'information si diffuse sur l'ensemble de la phrase que la distinction entre principale et subordonnée et la thématisation que manifeste normalement cette distinction deviennent floues, ce qui permet même d'intervertir les propositions sans que le texte paraisse en souffrir (je reviens sur cette particularité plus bas):

(10b) Today in Alton, Texas, investigators tried to determine [...] a school-bus into a waterfilled pit, as the inhabitants prepared to $[\ldots]$ the accident.

1.4. Cette équivalence dans l'expression de la temporalité entre as et alors que demeure toutefois limitée, ne serait-ce que stylistiquement. Si as s'emploie couramment dans ce sens temporel pur, il n'en est pas de même de alors que, dont le sens temporel est mis en marge par Le Bon Usage ${ }^{4}$, et cité comme «vieilli» par les Robert, «littéraire» par le Grand Dictionnaire encyclopédique Larousse. Le Grand Larousse de la langue française et le Trésor de la langue française sont heureusement plus tolérants, et en fait plus proches de l'usage contemporain, soutenu mais non point nécessairement littéraire, qui ne s'oppose pas formellement à l'emploi d'un alors que temporel restreint. Il n'en reste pas moins que, dans la majorité des cas, et en particulier lorsqu'il suit la principale, alors que affiche un sémantisme axé sur les notions d'opposition (exclusive ou non), de contraste adversatif (Robert), correspondant selon les cas à when, while ou whereas, sémantisme que je qualifierais de centrifuge, et en tout cas diamétralement opposé à celui de as, auquel je reviens ... maintenant.

2.0. L'emploi adversatif d'alors que, s'il s'inscrit souvent naturellement dans le synchronique, n'y est pas nécessairement lié: on peut opposer deux faits ou événements appartenant à deux moments historiques différents. Au contraire, les autres valeurs logiques de as continuent d'une manière ou d'une autre de satisfaire la condition d'étroite corrélation (chrono)logique entre les propositions (effet de zoom) qui caractérise déjà son sens temporel. Il est évident que, pour le traducteur ou le bilingue francophone, l'ignorance ou le mépris de cette divergence radicale des valeurs logico-syntaxiques respectives de ces conjonctions et l'alignement sur le modèle anglais aboutissent nécessairement à une infraction grave aux règles de la logique structurale du français international, du français langue autonome. Tous les cas de figure discutés ci-dessous donnent lieu au Canada 
français à des anglicismes, du fait de l'emploi abusif de alors que correspondant à telle valeur idiomatique de as. Il faut distinguer deux orientations principales, selon qu'un élément de causalité est présent ou non:

\subsubsection{La proposition introduite par as, crypto-complément direct : \\ Comparons l'exemple (1) avec:}

(11) The crowd cheered as the guest of honor arrived.

Là encore, $a s=$ when et introduit une action trop ponctuelle pour permettre de traduire as par alors que. Quand/lorsque conviendrait, mais il faut noter une différence significative entre (1) et (9), qui est que to disperse est intransitif, alors que to cheer est - psychologiquement, sémantiquement, sinon toujours syntaxiquement - transitif. Le fait que le complément d'objet puisse être sous-entendu (comme c'est très souvent le cas avec des verbes comme to eat, to like, to read, to watch, ... ) ne change rien à la relation d'actance qui caractérise ce verbe. Ne convient-il pas de voir dans la proposition introduite pas as le complément d'objet, quelque peu bâtard certes, du verbe, fonctionnellement équivalent à (... cheered) the guest of honor's arrival. Plutôt qu'un exemple fabriqué de toutes pièces, examinons celui-ci, emprunté à la presse écrite anglophone canadienne:

(12a) Thousands watch as doomed planes plunge into the lake.

Le présent historique neutralise les rapports aspectuels; transposée au passé, cette phrase deviendrait :

(12b) Thousands watched/were watching as doomed planes plunged into the lake.

(Aspect duratif dans la principale, ponctuel dans la subordonnée introduite par as (ou du moins d'une durée contenue dans celle de la principale), l'inverse donc du cas type de [1.1].)

Ce qui est particulier à ce modèle, c'est que les deux propositions peuvent échanger leur fonction syntaxique, basculer autour de as, sans que le noyau dur de l'information s'en trouve altéré de façon significative :

(12c) Planes crash into the lake as thousands watch $[\ldots]$

Phénomène de permutation tout à fait remarquable. Le principe de permutation est bien établi dans les cas de deux éléments syntaxiquement homologues et pouvant chacun exister de façon autonome:

- Pierre et Paul sont venus = Paul et Pierre sont venus $=$ Pierre est venu + Paul est venu.

- XX lit (ce)pendant que / et / tandis que / alors que (adversatif) YY regarde la télé... = YY regarde la télé (ce)pendant que / et / alors que / tandis que XX lit (angl. while/whereas)

C'est l'isomorphisme syntaxique et logique des deux éléments en cause qui rend cette opération possible. Celle-ci est d'ailleurs bien plutôt l'exception que la règle, en raison de la multiplicité des contraintes. Le as qui nous intéresse ici a ceci de particulier qu'il permet la permutation, bien que les faits décrits dans une des propositions soient endogènes - et non symétriques - à l'autre. Le verbe clé, to watch, est un verbe de perception, donc naturellement transitif ${ }^{5}$. Il existe entre les deux faits décrits une complémentarité qui s'apparente au lien entre le sujet et le complément direct, ou encore à l'alternative actif-passif des verbes transitifs: thousands watch crash/crash is watched by thousands, qui actualise (diathèse, thématisation) la constante des relations d'actance sous-jacente ${ }^{6}$. 
Le choix entre les deux constructions (12a) et (12c) est une question de thématisation, de focalisation, question mineure dans le cas d'une manchette de journal, sans contexte préalable, où donc tout est possible en l'absence des préconditions imposées par le discours. Il n'entraîne pas de changement à la réalité objective ou au rapport intime entre le fait brut et sa perception (voir note 6). Le rapport aspectuel et logique qui relie les deux propositions ne correspond en rien au sémantisme, temporel ou adversatif, de alors que; en revanche, un contexte tel que :

(12d) Planes plunge into lake [...] while thousands were having a picnic along the shore / as thousands were sailing and boating in the area,

qui respecte les critères aspectuels définis en (1.1), qui marque certes la proximité dans le temps et l'espace des deux faits rapportés (mais dont chacun est indépendant de l'autre, intransitif), et qui établit, surtout avec as, un lien logique discret à saveur de paradoxe (insouciance, inconscience de l'imminence de la catastrophe, la mort qui peut tomber du ciel à n'importe quel moment), se prêterait, lui, à une traduction de la conjonction par alors que.

Si dans (12a) la tension sémantique entre les deux propositions est si forte (d'où la possibilité de l'inversion, les deux faces nécessairement complémentaires d'une médaille), c'est qu'elle est du même ordre que celle qui relie le verbe transitif à son complément. Certes, le complément d'un verbe transitif, là où il est très vague et/ou contextuellement défini, peut être sous-entendu dans le texte, mais il ne peut pas l'être dans le message. En fait, il esț clair qu'ici, même si elle n'était pas au programme, la catastrophe, fortuitement, devient l'«objet», le «clou» tragique du spectacle, si bien que (12a) est, pour l'essentiel, l'équivalent idiomatique de :

(12e) Thousands watch planes plunge into lake ${ }^{7}$.

Alors que étant hors de question, restent au français les constructions:

(12e') Des milliers de personnes voient [les] avions [...] plonger dans le lac, ou encore :

(12c') Deux avions $[\ldots]$ lac sous les yeux de milliers de spectateurs (horrifiés...)

ou

(12") Collision entre deux avions au cours d'une démonstration: un mort, un blessé. Des milliers de spectateurs ont assisté à [...] /été témoins de [...] la tragédie.

Seule une différence théorique, et que je tiens en fait ici pour tout à fait secondaire, de focalisation sépare de même «la catastrophe s'est produite sous les yeux de milliers de spectateurs horrifiés» et «des milliers de spectateurs ont assisté horrifiés à la catastrophe / ont été témoins du drame ${ }^{8}$ ». La paraphrase n'altère en rien l'équivalence sémantique profonde.

2.1.2. As-clause en tant que contexte d'une principale endogène (c'est-à-dire qui rapporte un élément contributif, constitutif du contexte général).

Il ne saurait être question de causalité ici non plus, le rapport logique entre les deux propositions étant plutôt, comme pour la synecdoque, celui qui existe entre la partie (proposition principale) et le tout (proposition introduite par as) :

(13a) Gretzky scores hat-trick (last night) as L. A. blasts Detroit 10-2.

Cette construction rend l'idée de Most Valuable Player (la vedette/l'étoile du match), titre décerné exclusivement à un joueur de l'équipe victorieuse. De même : 
(14) Two years ago today, So-and-So pitched the only perfect game in World Series history, as the Purple Birds (bien évidemment l'equipe de So-and-So) defeated the Lowly Worms on their way to winning the Series.

On dirait tout aussi bien, toujours dans le moule endogène, même si la contribution est négative :

(15) $X$ fails to hit as the ZZZ's (X's team) drop 5th in a row/extend winless streak [...]/winless streak continues.

Le rapport direct et étroit établi entre les deux faits rapportés permet ici aussi la permutation des propositions ${ }^{9}$, si bien que l'on peut également dire ${ }^{10}$, allant cette fois du produit global à la composante la plus marquante :

(13b) L. A. blasts Detroit (last night) as Gretzky scores hat trick $(=\ldots$ with Gretzky scoring hat trick)

Ce jeu de bascule n'est pas sans rappeler, outre les constructions décrites précédemment (voir note 6), l'alternative que permettent des verbes tels que to result ou profiter, dont la polarité peut être inversée tout aussi facilement d'un simple coup de préposition ( $X$ resulted in $Y=Y$ resulted from $X$ ). Pareille symétrie ne se conçoit que lorsque les deux éléments en cause sont perçus comme étant virtuellement sur le même plan en fait de valeur informative; en d'autres termes, dans le cas présent comme dans (1.2), les oppositions thème-rhème/propos et information connue/information nouvelle sont neutralisées, les présuppositions suspendues... La phrase fait un tout, qui repose sur une table rase informationnelle et qui répond à une question générale (What's new?), à la différence des phrases contenant des propositions à information nouvelle gouvernées par since ou because, et à un moindre degré while. La construction donne l'apparence de la subordination, alors qu'en fait elle intègre deux événements dont l'un fait partie intégrante de l'autre (endogénie).

2.1.3. C'est à cette endogénie qu'il convient d'attribuer aussi l'usage, très proche du précédent, que l'anglais fait de as pour introduire moins une subordonnée adverbiale de temps, extérieure à la principale, qu'une apposition qui reprend en la développant, en l'agrandissant (effet de zoom), un ou plusieurs des compléments de la principale, et répond ainsi à la question How is that? / in what way? à propos de la principale. Le cas le plus fréquent, déjà signalé par SCFA ${ }^{11}$ à propos de when, sinon de as, est celui où la conjonction prend un complément de la principale, et tout particulièrement un complément circonstanciel de temps, comme antécédent de la proposition qu'elle gouverne. Par exemple, (13a) et (13b) peuvent s'employer avec ou sans last night, mais s'il est omis, c'est uniquement parce que le contexte permet implicitement la référence à last night; l'adverbe ne précède pas nécessairement immédiatement le as; $c f .(9 \mathrm{a}),(10 \mathrm{a})$ et $(14)$, où last week, today, et two years ago respectivement ne sont pas en position d'antécédents immédiats de as, mais il est toujours possible de les y mettre, et c'est en fait le cas le plus fréquent (c'est moi qui souligne, pour faire ressortir le rapport étroit - temporel, mais aussi logique, justificatif, le tout sous forme d'apposition à un today qui est Boxing day - qui existe entre l'adverbe et la subordonnée):

(16) There were line-ups in many centers today as shoppers vied for Boxing day bargains.

$\mathrm{Si}$, dans les exemples déjà cités, l'antécédent temporel peut être éloigné, voire sousentendu (apposition faible), il en est d'autres (apposition forte) où sa présence explicite est une condition nécessaire à l'emploi de la proposition subordonnée introduite par as: 
(17) The World Junior Hockey Championships open Saturday in Moscow as Canada plays Sweden.

En fait, l'antécédent de cette construction appositive est le plus souvent mixte: c'est tout le complexe adverbial spatio-temporel Saturday in Moscow qui étaie la subordonnée, encore que l'élément temporel soit l'élément dominant. Dans (16), c'est le complexe line-ups (...) today qui constitue l'antécédent. Dans d'autres cas, l'élément clé est le nominal, l'élément adverbial temps passant alors à l'arrière-plan et pouvant même être sous-entendu (c'est toujours moi qui souligne) :

(18) The California police are going on a manhunt (today) as they try to recapture escaped convict $X$...

Au lieu qu'elle décrive extrinsèquement les circonstances de l'événement décrit dans la principale, sur le mode de la proposition adverbiale typique, la proposition introduite par as développe le nom manhunt de la principale, comme le ferait une proposition adjectivale/relative (une chasse à l'homme au cours de laquelle...). Ce rôle est encore plus évident dans l'exemple suivant, où la reprise de l'antécédent touche au pléonasme (c'est toujours moi qui souligne):

(19) (...) the careful reader [of the U.S. press] will discern [the doubts expressed by the German journalists] in the reports of the continuing investigation, as suspects alleged to have Syrian and other connections are investigated ${ }^{12}$.

Cette intégration des propositions qui encadrent as dans les cas ci-dessus se retrouve dans les constructions suivantes, où as exprime ce rapport logique étroit, centripète, qu'est la causalité.

2.2. As et l'expression de la causalité, sous une forme ou sous une autre: indirect and direct reason relationship... We subsume under clauses of REASON several types of subordinate clauses that convey basic similarities of relationship to their matrix clause. For all types there is generally a temporal sequence such that the situation in the subordinate clause precedes in time that of the matrix clause ${ }^{13}$.

2.2.1. Temporel + raison logique: ... (b) Reason and consequence: the construction expresses the speaker's inference of a connection ${ }^{14}$. As a une fonction explicative; elle introduit un justificatif de l'énoncé de la principale; la temporalité est conditionnée par le rapport logique posé :

(20) *La population est priée de ne pas fumer dans la forêt, alors que la moindre étincelle risque de provoquer une catastrophe (contexte coextensif/simultanéité).

Cet anglicisme témoigne du fait que as s'emploierait tout naturellement en anglais dans pareil contexte (correspondant au modèle $[\mathrm{A}$, as $\mathrm{B}]=[$ I can say A because $\mathrm{B}]$ ). Il ajoute à la temporalité un élément déterminant de causalité oblique, et, à ce titre se rapproche de because:

As a conjunction, one sense of as is because: As she was bored, Sue left the room. As also has an equally common use in the sense while, when: As the parade passed by, the crowd cheered and applauded. The two senses sometimes result in ambiguity: As the gates were closed, he walked away. (When? Because?)... (The Random House Dictionary of the English Language) et, davantage encore, de since:

It is a testimony of the close and obvious connection between reason and temporal sequence that as and since are conjunctions of time as well as of cause. This dual function can give rise to ambiguity: 
As he was standing near the door, he could hear the conversation in the kitchen. [ Since he was standing near the door...» or "While he was standing near the door»... $\left.{ }^{15}\right]$

Il existe toutefois entre since et le as qui nous intéresse ici des différences significatives: (i) la as-clause peut introduire de l'information nouvelle; c'est pourquoi as, tout comme because, suit généralement la principale, alors que since la précède; (ii) as répond simultanément aux questions why? et when?, ou peut-être plus justement why then / why now? ; c'est-à-dire qu'à la causalité, il associe toujours, dans le même mouvement, la temporalité - parfois même, comme nous l'avons déjà noté dans (2.1.3), sous une forme qui tend à faire de la proposition introduite par as une apposition: the public is requested not to use open fires in the park this week-end, as (= a time at which) the slightest spark... Since a également une dimension temporelle, mais le risque d'ambiguiité est pratiquement nul, car le contexte, et plus précisément le temps des verbes, varie généralement selon que since est causal ou temporel, car il n'est pas question pour lui de combiner les deux ${ }^{16}$.

Plutôt que since, l'anglais pourrait aussi employer as long as, ou given that (at this/ that time), ou «(in somewhat formal style) for ${ }^{17} \gg$. Le français aurait le choix entre car, qui rend explicitement la relation causale et laisse implicite la relation temporelle (pour une équivalence qui traduise la bidimensionnalité de $a s$, on pourrait facultativement ajouter maintenant, ou combiner $\grave{a}$ un moment $/ \grave{a}$ une époque... avec vu que lattendu $q u e$ ), et, en coordination, ... forêt; en effet, (actuellement/la situation actuelle est telle que)...

Autres exemples, en anglais cette fois :

(21) Those two runs proved to be the difference / X's solo blast in the 3rd inning proved to be the winning run as Baltimore eventually managed to squeeze by Washington 3-2.

As introduit les circonstances, le contexte général, le fait principal, the bottom line en quelque sorte, mais aussi la justification a posteriori de l'affirmation de la principale touchant the winning run/the difference dans le contexte en question. Pour rendre cette justification, le français emploierait la coordination pure et simple à l'aide de et ou, après un; /:, une seconde proposition indépendante commençant par en effet/à preuve. La phrase relie ainsi plus étroitement la chronologie des événements à la réaction psychologique du spectateur (et Baltimore finissait par remporter de justesse la victoire sur Washington 3-2, ou car / en effet B allait / devait finir par l'emporter...). Enfin :

(22) The Maple Leafs ended up with a pretty good scoring chance as the Bruins defense got caught up the ice (intersection de la simultanéité et de la causalité).

Tant dans (20) (ou, du moins, son équivalent anglais) que dans (21) et (22), while serait exclu parce que trop duratif et pas assez causal, cependant que when serait possible, mais uniquement dans un sens temporel (aspect fréquentatif, en raison de l'ambiguitté du présent) pour (20); pour (22), when exprimerait la causalité de fait ( $c f$. infra). As, lui, pose un rapport logique, explicatif, entre l'affirmation de pretty good scoring chance, interprétation des faits par le commentateur, et le fait que the Bruins defense got caught up the ice, en vertu de la règle empirique selon laquelle whenever the defense gets caught up the ice, the opposing team's offense has a numerical advantage over the defense, hence, a good scoring opportunity.

2.2.2. Temporalité + causalité de fait : (a) Cause and effect : the construction expresses the perception of an inherent objective connection in the real world ${ }^{18}$. 
Comme il sied à un rapport de causalité effective, la temporalité, de simultanée, se fait (immédiatement) consécutive. Par ailleurs, les remarques précédentes concernant la portée de as vs while vs since vs when demeurent valables: while n'est pas assez causal, since l'est trop exclusivement (ou s'il est temporel, c'est dans un sens qui n'est pas approprié au contexte), et when, auquel l'anglais attribue aussi naturellement une fonction causale, ne peut s'employer (dans (24), par exemple) que lorsque l'événement décrit dans la proposition qu'il gouverne peut être perçu comme ponctuel, comme c'est objectivement le cas pour une explosion ${ }^{19}$ ou un accident de voiture. Certes, un typhon n'est pas un phénomène naturel ponctuel comme l'est la foudre, mais l'emploi de hit le pose fonctionnellement comme tel. Le français met généralement l'accent sur ce rapport de causalité, soit de façon explicite ${ }^{19}$.

(25') Le recul de la violence (...) permet aux autorités colombiennes de lever (...) soit par juxtaposition des faits dans l'ordre (chrono)logique cause $\rightarrow$ effet :

(25") Diminution de la violence... : les autorités colombiennes lèvent le couvre-feu...

(23') La grève du personnel hospitalier du Québec s'étend : les hôpitaux (sont) au bord de la crise.

3. Conclusion: L'analyse qui précède illustre la remarquable polyvalence et flexibilité de la conjonction as, même circonscrite à ses emplois non comparatifs. Il n'est pas rare de voir l'usage brouiller les distinctions que ma classification tente d'établir au profit d'ambiguïtés, spontanées ou calculées, comme l'anglais sait si bien les cultiver ( $c f$. le cas classique du degré zéro de l'article, défini (générique) ou indéfini, dans le premier paragraphe de l'article 1 de la Résolution 242 de l'ONU, qui parle du «withdrawal of Israeli armed forces from territories occupied in the recent conflict»), et auxquelles le français, par souci traditionnel de précision ou par impuissance, est absolument réfractaire. C'est ainsi que les deux premiers paragraphes de l'article du Toronto Star intitulé

(26) Sikh troops mutiny as temple toll climb

nous apprennent que:

Mutinies by Sikhs in the Indian Army spread yesterday as violent reaction to the military assault on Amritsar's Golden Temple widened to other parts of the country.

Between 700 and 1,000 people were reported killed in the attack on the Sikhs' holiest shrine in Punjab state last week. However, in a report from Amritsar, Associated Press said the final death toll could be as high as $2,000 \ldots$

Il se trouve que le premier paragraphe illustre l'emploi appositif de as, axé sur l'adverbial de temps et flirtant même avec le pléonasme (spread (...) widened to other parts of the country) - emploi décrit dans 2.1.3. et dans 2.2.1, qui semble répondre à la question implicite portant sur la principale: How was that? / Can you elaborate on that statement? / What exactly do you mean by that statement? Mais, pour revenir à l'interprétation du as de l'exemple (26), ces deux paragraphes nous permettent d'établir que:

(i) as relie les deux propositions dans un rapport temporel, de simultanéité : tout se situe dans le cadre de yesterday (as = alors que /(ce)pendant que...?);

(ii) en même temps, il postule un lien de causalité, qui implique une temporalité de type consécutif et non point strictement simultané: violent reaction; c'est le modèle que 
j'ai décrit dans 2.2.2, avec toute l'ambiguïté qu'il comporte (parce que/puisque/vu que...?);

(iii) à l'évolution dans le temps de l'élément causal (un bilan qui, de 700-1 000 victimes, risque d'atteindre le chiffre de 2000 ) correspond, par voie de conséquence, une évolution parallèle de l'effet; la mutinerie n'est pas éruption ponctuelle mais boule de neige: mutinies (...) spread (...) widened to other parts of the country. (au fur et à mesure que...?).

De même :

(27) The Syrians said they would withdraw as the Israeli withdrew

où as signifie tout à la fois si (et seulement si), quand, aussitôt que et aussi longtemps que, à mesure que et dans la mesure où...

S'il ne veut pas multiplier les conjonctions de façon à reproduire fidèlement le sémantisme complexe de as, le français, prisonnier de son corset logico-syntaxique, est obligé de s'en tenir à l'élément le plus pointu, s'en remettant au contexte pour le reste. Ce faisant, il risque toutefois de faire tomber le lecteur de Charybde en Scylla, d'une ambiguïté souvent plus théorique que réelle à une précision réductionniste qui souvent ne parvient même pas à éliminer l'ambiguïté, dans la mesure où le contexte auquel faire appel est souvent sibyllin et équivoque. Si bien que ce as que nous accusions précédemment de fomenter l'ambiguïté s'avère en fait un instrument logico-syntaxique d'une puissance et d'une adaptabilité extrêmes, capable, en toute simplicité (une syllabe, deux graphèmes, deux phonèmes, il est difficile d'aller plus loin dans la simplicité), de manger simultanément à plusieurs rateliers, d'exprimer toute une gamme de sémantismes complémentaires. Conjonction caméléon, outil polyvalent permettant de raccorder syntaxiquement deux propositions logiquement solidaires, à valeur adverbiale (temporelle-causale) aussi bien que nominale (le crypto-complément d'objet de 2.1.1), voire adjectivale (appositive, 2.1.3), as fait penser, par sa simplicité et sa souplesse d'emploi, au que passe-partout du français, littéraire ou familier, selon le $\operatorname{cas}^{20}$. Pour le traducteur en tout cas, la leçon est claire : il doit éviter de rendre mécaniquement et aveuglément un terme aussi idiomatique, aussi fuyant, mais fondamentalement centripète, par un alors que naturellement centrifuge qui risque fort de trahir le message et, plus généralement, d'introduire un élément important de confusion dans le champ logico-syntaxique du français. La «clarté française» tant vantée depuis Rivarol n'est peut-être qu'un mythe, mais «le mythe de la clarté a du moins l'avantage de produire une éthique de la clarté ${ }^{21}$ ». Il serait quand même dommage de lui substituer une éthique de la facilité 22 .

\section{NOTES}

1. C'est-à-dire qu'au passé du moins — car le présent occulte les rapports aspectuels - cet alors que temporel ne peut être suivi que par une forme imperfective du verbe, par exemple l'imparfait, jamais par le passé simple ou le passé composé.

2. On peut également considérer cette construction comme la subordination d'un rapport qui, sur le plan de la coordination, serait marqué au moyen de l'adverbe (c'est) alors (que)/at that very moment - then/c'est le moment qu'a choisi (...) pour (...).

3. Stylistique comparée du français et de l'anglais, $\$ 87,1$ ).

4. «La proposition adverbiale de temps, 1081 ; Mots de liaison... (b) les deux faits sont simultanés : Comme, pendant que, tandis [tãdi] que, en même temps que, tant que, outre alors que, qui implique d' habitude une nuance d'opposition.» (C'est moi qui souligne.)

5. Si la transitivité de to cheer (exemple [11]) était discutable, les dictionnaires anglais reconnaissant à ce verbe un emploi intransitif au sens de to utter a shout of applause or triumph (Webster), il n'en va pas de même de to watch, incontestablement transitif, selon les mêmes dictionnaires, dans le sens qui nous intéresse ici : (...) to look at : OBSERVE (...) ou to look on at ... (ibid.). 
6. Cette commutativité rappelle aussi les cas d'interversion des actants du type le blé manquait à l'armée l'armée manquait de blé (Éléments de syntaxe structurale, p. 286), ou bees swarmed in the garden - the garden was swarming with bees, ou enfin, dans une perspective interlinguale, I miss you - vous me manquez; autant de cas où les deux actants sont sémantiquement intimement liés, indissociables, et où les critères habituels de détermination de l'ordre séquentiel (sujet animé, rapport de causalité...) sont suspendus, à tel point que le choix du sujet grammatical, hors situation, devient aléatoire. En structure sémantique profonde, il est probable que la fonction dont serait dérivé le verbe transitif de l'énoncé de surface aurait nécessairement deux arguments (bivalence), aussi essentiels l'un que l'autre - ce qui n'empêche nullement que celui (quel qu'il soit) des actants qui les lexicaliseront qui n'aura pas été retenu pour occuper la position de sujet grammatical, puisse être sous-entendu en structure de surface: «ce chien mord./Le facteur a été mordu. (...) du point de vue structural (...) le sujet est un complément comme les autres» (ibid., p. 109.)

D'une manière générale, il s'agit de ces constructions qu'unit ce que J. Lyons, traitant de la sémantique lexicale, appelle un rapport de converseness :

(...) the lexical substitution of one term for the corresponding antonym or converse is associated with a syntactic transformation which permutes the noun-phrase, $N P_{1}$ and $N P_{2}$ [il donne comme exemples buy - sell et husband-wife], and also carries out certain other "automatic» changes in the selection of the appropriate preposition... It may also be observed that this «permutational feature» is also characteristic of the relationship between corresponding active and passive sentences...

Other lexical items are «permutationally» related in the same way as converse terms, although they do not imply one another. For example, $N P_{1}$ asked $\left(N P_{2}\right) \ldots$ «expects», rather than implies, $N P_{2}$ answered $\left(N P_{1}\right) \ldots ;$ and $N P_{2}$ answered $\left(N P_{1}\right) \ldots$ presupposes $N P_{1}$ asked $\left(N P_{2}\right) \ldots$ «Expectancy» and "presupposition» of this kind are ordered with respect to temporal sequence: this is not so, it should be noted, in the case of such converse terms as give and receive. (Introduction to Theoretical Linguistics, p. 467-469.)

7. Je ne prétends pas gommer la nuance sémantique qui existe, théoriquement du moins, entre ces deux constructions : lorsque le complément de watch / listen prend la forme d'une proposition introduite par as, il en résulte une certaine disjonction, l'introduction d'une certaine distance entre l'action/la perception exprimée par le verbe principal et l'objet final de cette action/perception. Dans le cas de (12a), le complément de watch est ajouté comme après coup, après des points de suspension, pour ménager le suspense ou mettre davantage en relief chacun des deux éléments d'information, sous forme de la proposition gouvernée par as, laquelle, à la différence de la proposition infinitive de (12e), est potentiellement indépendante et se prête à la disjonction. Cela étant dit, dans la pratique, cette nuance est bien loin d'être systématiquement respectée. 8. Autre exemple d'actualité :

(12f) Scientists watch as Voyager races towards Saturn (= Voyager races towards Saturn as scientists watch $=$

Scientists watch Voyager race towards Saturn $=$ Scientists watch $/$ follow Voyager's race towards Saturn).

SCFA nous proposait déjà un exemple comparable, où figure un to cheer tout aussi transitif que to watch cidessus (à la difference de People whispered as the troups marched by, par exemple):

$(12 \mathrm{~g})$ People cheered as the troups marched by:

Les gens ont applaudi sur le passage des troupes. $(\$ 87$ )

On pourrait tout aussi bien dire :

(12h) People cheered the marching of the troups / the march-past, ou the troups marched by as the people cheered,

ou, en français cette fois,

(12h') les gens ont applaudi le défilé militaire,

ou

les troupes ont défilé sous les ovations de la foule.

9. Cf. définition de la synecdoque (c'est moi qui souligne) : «Figure de thétorique qui consiste à prendre (...) l'espèce pour le genre, la partie pour le tout, le singulier pour le pluriel ou inversement (...)» (Petit Robert.)

10. Quoique moins fréquemment; en fait, cette construction ne faisait pas l'unanimité parmi les informateurs anglophones que j'ai consultés. Son utilisation dans le jargon journalistique s'explique peut-être comme l'expression atténuée de la causalité (cf. 2.2).

11. §93. 3) Étoffement des conjonctions : a) conjonctions en appositions: «Ni notre quand ni notre où ne peuvent, en apposition, s'employer sans un étoffement». Cet étoffement peut d'ailleurs prendre des formes variées, par exemple :

Derrière le cosmonaute soviétique (le recordman du monde Sergueï Bubka), le niveau de la spécialité

(le saut à la perche) n'a guère évolué depuis qu'en 1985 il (- Pierre Quinon, champion olympique

en 1984) tutoyait régulièrement les barres à 5,90 mètres. (Le Monde, 21-22/8/88, p. 7.)

(... pole vaulting has not progressed much since 1985, when Quinon routinely used to clear 19 feet) 
qui équivaut à «... depuis qu'en 85 , année pendant laquelle...», selon le modèle de SCFA. La conjonction depuis que englobe (comme pourrait d'ailleurs le faire la conjonction since, à laquelle l'usage semble toutefois préférer l'autre formule) la préposition since et la conjonction when, remplaçant ainsi la «conjonction en apposition» par une proposition subordonnée de temps en bonne et due forme. Ou encore :

La crise de confiance entre les pasdarans et le pouvoir semble avoir commencé en avril 1987 a l'occasion des élections législatives qui opposèrent le clan des «radicaux» (...) aux «conservateurs» de la droite religieuse traditionaliste. (Ibid., p. 5)

(the crisis [...] apparently started at the time of the «parliamentary» elections, back in April 1987, when [the two clans] confronted each other);

cette fois, la solution est de type proposition relative.

12. Covert Action Information Bulletin, $\mathrm{n}^{\circ} 26$, Summer 1986, p. 16.

13. A Comprehensive Grammar of the English Language, p. 1103 et ss.

14. Ibid., p. 1104.

15. Ibid., p. 1105.

16. Il est d'ailleurs révélateur que la glose de la citation ci-dessus fasse appel à since (comme le Random House Dictionary le fait à because, voir 2.2.1, supra) pour désambiguïser le texte. En effet, since n'est ambigu que lorsqu'il exprime une cause ponctuelle, car il recoupe alors l'aspect ponctuel/inchoatif qui caractérise le since temporel: Since he has left, ne peut être que causal, d'emblée, car le temps du verbe dénote un état. En revanche, Since he left... s'annonce comme potentiellement ambigu, mais là encore le temps du verbe principal dissipe normalement l'ambiguité ; dans la phrase Since he left (= since July 14th, par ex.), I've been very sad, la proposition subordonné marque le point de départ de la situation décrite par la principale; tandis que dans since he left (* since July 14 th ), I'll have to manage on my own, since est manifestement causal. Tout au plus peut-on parler d'ambiguité dans un contexte tel que since he left, I have to do everything myself, encore qu'avec un since vraiment temporel (= since July $14 t h)$, il faudrait dire..., I've had to do everything myself.

17. A Comprehensive Grammar of Contemporary English Usage, p. 1104.

18. Ibid., p. 1103.

19. «Three men were killed when a tank blew up.» Il ne s'agit pas d'une coïncidence mais d'un rapport de cause à effet. Disons donc: (...) l'explosion d'un réservoir fit [ou fait, présent de narration] trois victimes» (SCFA, § 155).

20. «(...) [Que] s'emploie, surtout dans la langue familière, à la place de diverses autres conjonctions ou locutions conjonctives : (...)

Ces emplois sont particulièrement fréquents dans le Midi, où que équivaut notamment à car du français commun: Je ne peux pas courir, QUE je suis trop vieux. - Parfois, la valeur du que n'est pas facile a expliciter...» (Le Bon Usage, § 1028.)

«(...) que est souvent, comme dit Brunot, une "conjonction universelle", apte à jouer, depuis toujours, dans la langue commune ou populaire, et parfois très hardiment, les rôles les plus divers...» (Le Bon Usage, $11^{e}$ édition, $1980, \$ 2501$.)

Nous avons déjà rencontré cette valeur de $a s=$ for (2.2.1). Or, le for en question, conjonction de coordination (angl.) est normalement équivalent au français car, lequel, nous venons de le voir, dans le Midi en tout cas, est parfois remplacé par que. D'où nous pourrions conclure que as = que. Génêralisation manifestement trop grossière pour être universellement correcte. L' 'universalité» dont parle Brunot est purement relative et se caractérise en fait par la multiplication des contraintes, qui font de chacun des divers $q u e$, comme de chaque as en anglais, une chausse trappe idiomatique. Notons toutefois que, dans la perspective qui nous intéresse et dans sa seule fonction de conjonction, que est capable, entre autres :

(a) d'exprimer «l'incidence d'un fait instantané par rapport à un fait continu...

"La mort nous prend que nous sommes encore tout pleins de nos misères et de nos bonnes intentions." (Mme de SÉVIGNÉ)» (Le Grand Robert de la langue française, vol. 7, p. 945),

sens remarquablement proche de celui de as / alors que décrit dans 1.1.

(b) de souligner le fait saillant d'un événement :

«Pop. Même que (En tête d'une phrase à l'indicatif ou au conditionnel). Et même, bien plus...» (ibid., vol. 6, p. 355).

Au niveau de la langue populaire du moins, l'exemple (13b) pourrait donc se traduire ainsi: «Les Kings écrasent Détroit 10-2, même que Gretzky, il a marqué 3 buts.»

(c) «Dans des phrases interrogatives ou exclamatives, (d') indiquer la cause, non du fait exprimé par le verbe principal, mais de la demande ou de l'exclamation que ce fait a suscitées de la part du sujet parlant :

«Mais tu n'as pas faim, QUE tu ne finis pas tes huîtres ?» (BOURGET)» (Le Bon Usage, § 1083, Remarques. - 1.)

La logique de ce que n'est évidemment pas sans rappeler celle du as de 2.2.1.

21. Le Français et les siècles, p. 170. 
22. Comment interpréter en effet la phrase suivante, à moins évidemment de connaître l'anglais - mais alors, à quoi bon le français?

*Hier soir, au Forum, $\mathrm{X}$ a marqué 3 buts / a réussi le tour du chapeau alors que Montréal battait Toronto 4-1, alors que Boston [le grand rival de Montréal] devait se contenter d'un match nul 2-2 devant Vancouver et se retrouve de ce fait à quatre points de la tête de la division.

Comme il se doit, l'anglais emploie naturellement deux conjonctions différentes (as et while / whereas) pour exprimer deux fonctions logico-syntaxiques différentes. L'alignement aveugle de alors que sur as, alors que alors que est déjà l'équivalent de while / whereas, aboutit ici à la neutralisation de la différence de fonction, donc au non-sens.

\section{BIBLIOGRAPHIE}

Ouvrages généraux

Grand Dictionnaire encyclopédique Larousse (1982), 10 vol., Paris, Larousse.

Grand Larousse de la langue française (1971), 6 vol., Paris, Larousse.

Grand Robert de la langue française (1987), $2^{\mathrm{e}}$ édition, 9 vol., Paris, Éditions Le Robert.

Random House Dictionary of the English Language (1987), second edition, unabridged.

Trésor de la langue française (1972-1989) : Paris, Éditions du CNRS, 13 volumes parus.

Webster New Collegiate Dictionary, Merriam-Webster, 1981.

\section{Ouvrages spécialisés}

CHOMSKY, Noam (1986) : Covert Action Information Bulletin, $\mathrm{n}^{\circ} 26$.

GREVISSE, Maurice (1980) : Le Bon Usage, $11^{\mathrm{e}}$ édition, Paris-Gembloux, Duculot.

GREVISSE, Maurice (1988) : Le Bon Usage, $12^{\mathrm{e}}$ édition refondue par André Goosse, Paris-Gembloux, Duculot (sauf indication contraire, c'est cette édition qui est citee).

HAGĖGE, Claude (1987): Le Français et les siècles, Paris, Odile Jacob.

LYONS, John (1969) : Introduction to Theoretical Linguistics, Cambridge University Press.

QUIRK, Randolph, Sidney GREENBAUM, Geoffrey LEECH, and Jan SWARTVIK (1985) : A Comprehensive Grammar of Contemporary English Usage, New York, Longman.

TESNIÈRE, Lucien (1958) : Eléments de syntaxe structurale, Paris, Klincksieck.

VINAY, J.-P. et J. DARBELNET (1969) : La Stylistique comparée du français et de l'anglais, Paris, Didier (abreviation SCFA). 